\title{
Bulk viscosity of water in acoustic modal analysis and experi- ment
}

\author{
Jan Kůrečka ${ }^{1, \star}$, Vladimír Habán ${ }^{1, \star \star}$, and Daniel Himr ${ }^{1, \star \star \star}$ \\ ${ }^{1}$ Victor Kaplan Department of Fluid Engineering, Brno University of Technology, Technická 2896/2, Brno, 616 69, Czech Republic
}

\begin{abstract}
Bulk viscosity is an important factor in the damping properties of fluid systems and exhibits frequency dependent behaviour. A comparison between modal analysis in ANSYS Acoustics, custom code and experimental data is presented in this paper. The measured system consists of closed ended water-filled steel pipes of different lengths. The influence of a pipe wall, flanges on both ends and longitudinal waves in the structural part were included in measurement evaluation. Therefore, the obtained values of sound speed and bulk viscosity are parameters of the fluid. A numerical simulation was carried out only using fluid volume in a range of bulk viscosity. Damping characteristics in this range were compared to measured values. The results show a significant influence of sound speed and subsequently, the use of sound speed value regressed from experimental data yields a better fit between the measurement and the computation.
\end{abstract}

\section{Introduction}

Bulk viscosity is a parameter describing dissipation of energy in a fluid caused by compression and dilatation and can be defined as a resistance of fluid against change of volume. It is different from shear viscosity describing energy loss of flowing liquid. More information from studies focused on determination of bulk viscosity of different fluids is summarized in [2]. Previous studies show that bulk viscosity is frequency dependent[3].

Finite element method (FEM) acoustic analysis can be used to predict sound propagation, vibroacoustic problems, response of fluid system to excitation and other applications related to fluid acoustic [4], [5], [6]. Acoustic simulation applied to a design of a muffler, design of a damper of pressure pulsations and modification of industrial fan after failure are described in mentioned references. ANSYS acoustic simulation software is solving Navier-Stokes and continuity equations linearized and modified into wave equation. Analogically to degrees of freedom in mechanical solver the acoustic simulation solves degrees of freedom of acoustic pressure and velocity [7]. Aim of this contribution is to discuss how commonly used acoustic analysis software treats bulk viscosity and compare simulation to experiment and theory used in evaluation process.

\section{Experiment description}

The experimental system consists of pipe equipped with four sensors, two accelerometers and two pressure sensors

\footnotetext{
^e-mail: 133644@vutbr.cz

$\star \star$ e-mail: haban@fme.vutbr.cz

$\star \star \star$ e-mail: himr@fme.vutbr.cz
}

on each end of pipe as depicted in Fig. 1. The pipe is at one end connected to a pump and there is a ball valve in between that is used to close the pipe after pressurization. At the opposite end of pipe the pipe is connected to a valve used to remove trapped air from system.

The pipe was excited by hitting it with a hammer and pressure oscillations and accelerations were measured. Real and imaginary part of eigen value was determined by FFT. Real part was taken as width of resonance range at half amplitude.

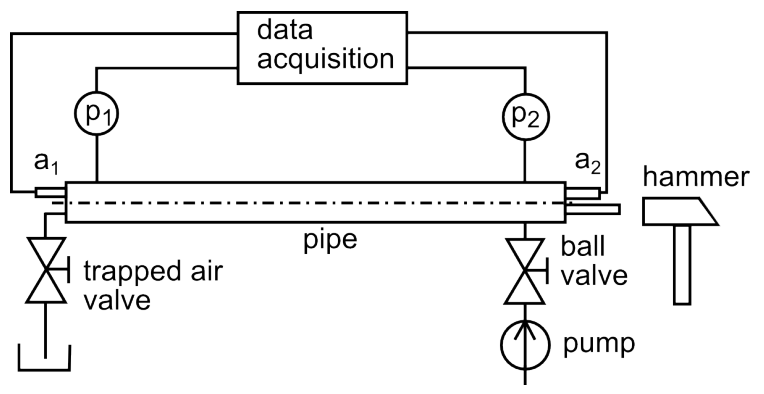

Fig. 1. Measurement system $\left(a_{1,2}\right.$ - accelerometers, $p_{1,2}$ - pressure sensors)

\subsection{Dry pipe experiment}

Before measurement of a water-filled pipe, it was necessary to determine material properties of structural part of the pipe. For this reason, experiment with an empty tube was performed. In the following part, theory behind evaluation of a measured data is described. 


\subsection{Transfer matrix and material properties of dry pipe}

There is a rod (assuming the situation is same for a pipe) from elastic material. Its length dimension is significantly bigger than its diameter, its cross-section is constant, cross-sections perpendicular to the rod axis remain planar even when the rod is deformed and the stress is equally distributed in a cross-section. Moment equation of a rod element can be written as:

$$
S_{p} \cdot \rho \frac{\partial^{2} x_{1}}{\partial t^{2}} \cdot d x=S_{p} \cdot \frac{\partial \sigma}{\partial x} \cdot d x
$$

It can be adapted into form describing a longitudal rod displacement (index 1 means direction along the length of the pipe or axial direction).

$$
\rho \frac{\partial^{2} x_{1}}{\partial t^{2}}=\frac{\partial \sigma}{\partial x}
$$

Subsequently the stress $\sigma$ can be expressed using Voigt-Kelvin material model.

$$
\sigma=E_{1} \cdot \frac{\partial x_{1}}{\partial x}+b_{1} \cdot \frac{\partial^{2} x_{1}}{\partial x \cdot \partial t}
$$

Combining Eq. (3) and Eq. (2) and minor modifications:

$$
\rho \cdot \frac{\partial^{2} x_{1}}{\partial t^{2}}=E_{1} \cdot \frac{\partial^{2} x_{1}}{\partial x^{2}}+b_{1} \cdot \frac{\partial^{3} x_{1}}{\partial x^{2} \cdot \partial t}
$$

Laplace transform of this equation and separation of differential on the right hand side:

$$
s^{2} \cdot \tilde{x}_{1} \cdot \frac{\rho}{\left(E_{1}+b_{1} \cdot s\right)}=\frac{\partial^{2} \tilde{x}_{1}}{\partial x^{2}}
$$

Using speed of sound $c_{0}$ formulated in Eq. (6) the moment equation can be further modified.

$$
\begin{aligned}
& \frac{1}{c_{0}^{2}}=\sqrt{\frac{\rho}{E_{1}}+b_{1} \cdot s} \\
& \frac{\partial^{2} \tilde{x}_{1}}{\partial x^{2}}=\frac{1}{c_{0}^{2}} \cdot s^{2} \cdot \tilde{x}_{1}
\end{aligned}
$$

Steady vibrations in axial direction formulated as a function:

$$
\tilde{x}_{1}=U(x) \cdot e^{i \cdot s \cdot t}
$$

Voigt-Kelvin model after Laplace transform:

$$
\tilde{F}=\tilde{S}_{p} \cdot\left(E_{1}+b_{1} \cdot s\right) \cdot \frac{\partial \tilde{x}_{1}}{\partial x}
$$

Solution of momentum equation with respect to steady vibrations formulation in Eq. (8) is:

$$
\tilde{x}_{1}(x)=C_{1} \cdot \cosh \left(\frac{s}{c_{0}} \cdot x\right)-C_{2} \cdot \sinh \left(\frac{s}{c_{0}} \cdot x\right)
$$
tion:

Derivation of this equation gives following formula-

$$
\frac{\partial \tilde{x}_{1}}{\partial x}=C_{2} \frac{s}{c_{0}} \cosh \left(\frac{s}{c_{0}} \cdot x\right)-C_{1} \frac{s}{c_{0}} \sinh \left(\frac{s}{c_{0}} \cdot x\right)
$$

Constants $C_{1}$ and $C_{2}$ can be determined from the boundary condition at $\mathrm{x}=0$ along with known Laplace images of force $\tilde{F}$ and displacement $\tilde{x}_{1}$ :

$$
\tilde{x}_{1}(x=0)=C_{1} \cdot \cosh (0)+C_{2} \cdot \sinh (0)
$$

Therefore, a Laplace image of displacement at start of pipe equals constant $C_{l}$ :

$$
\tilde{x}_{1}(x=0)=C_{1}
$$

To obtain constant $C_{2}$ equations describing V-K model after Laplace transformation Eq. (9) and derivation of momentum equation Eq. (11) can be used.

$$
\tilde{F}(x=0)=\tilde{S}_{p} \cdot\left(E_{1}+b_{1} \cdot s\right) \cdot \frac{s}{c_{0}} \cdot C_{2}
$$

Then second constant can be expressed in a following formulation:

$$
C_{2}=\frac{\tilde{F}}{\tilde{S}_{p} \cdot\left(E_{1}+b_{1} \cdot s\right) \cdot \frac{s}{c_{0}}}
$$

Finally, the matrix formulation can be written using the transform matrix $\mathbf{P}_{\mathrm{T}}$, describing vector $\mathbf{u}$ at any length of pipe.

$$
\mathbf{u}(x, s)=\mathbf{P}_{T}(x, s) \cdot \mathbf{u}(0, s)
$$

$$
\mathbf{u}=\left(\begin{array}{c}
\tilde{x}_{1} \\
\tilde{F}
\end{array}\right)
$$

$$
\mathbf{P}_{T}=\left(\begin{array}{cc}
\cosh \left(\frac{s}{c_{0}} \cdot x\right) & P_{T 12} \\
P_{T 21} & \cosh \left(\frac{s}{c_{0}} \cdot x\right)
\end{array}\right)
$$

Elements $P_{T 12}$ and $P_{T 21}$ are formulated in following equations.

$$
P_{T 12}=\frac{1}{\tilde{S}_{p} \cdot\left(E_{1}+b_{1} \cdot s\right) \cdot \frac{s}{c_{0}}} \cdot \sinh \left(\frac{s}{c_{0}} \cdot x\right)
$$

$$
P_{T 21}=\tilde{S}_{p} \cdot\left(E_{1}+b_{1} \cdot s\right) \cdot \frac{s}{c_{0}} \cdot \sinh \left(\frac{s}{c_{0}} \cdot x\right)
$$

Transfer matrices $\mathbf{P}_{10}$ and $\mathbf{P}_{23}$ were used to account for weight of flanges, connected hoses and valves on both ends (index 0 means start of pipe, index 3 end of pipe, 1 is position right after start of pipe, 2 analogical to 1 ).

$$
\mathbf{P}_{10}=\mathbf{P}_{23}=\left(\begin{array}{cc}
1 & 0 \\
M \cdot s^{2} & 1
\end{array}\right)
$$


Equation in matrix form using above mentioned transfer matrices:

$$
\left(\begin{array}{c}
\tilde{x}_{1_{3}} \\
\tilde{F}_{3}
\end{array}\right)=\mathbf{P}_{10} \cdot \mathbf{P}_{T} \cdot \mathbf{P}_{23}\left(\begin{array}{c}
\tilde{x}_{1_{0}} \\
\tilde{F}_{0}
\end{array}\right)
$$

With boundary conditions, Eq.(23), (24) and a few modifications, the final equation Eq.(25) used to obtain material properties of the tube $\left(E_{l}\right.$ and $\left.b_{1}\right)$ is:

$$
\begin{aligned}
& S_{0}=0 \\
& S_{3}=0
\end{aligned}
$$

$$
\left(\begin{array}{l}
0 \\
0 \\
0 \\
0
\end{array}\right)=\left(\begin{array}{cccc}
0 & 1 & 0 & 0 \\
0 & 0 & 0 & 1 \\
P_{T f 31} & P_{T 12} & -1 & 0 \\
P_{T f 41} & P_{T f 42} & 0 & -1
\end{array}\right) \cdot\left(\begin{array}{c}
\tilde{x}_{1_{0}} \\
\tilde{F}_{0} \\
\tilde{x}_{1_{3}} \\
\tilde{F}_{3}
\end{array}\right)
$$

And elements of this matrix are written in Eq. 26, 27 and 28 .

$$
\begin{gathered}
P_{T f 31}=P_{T 11}+P_{T 12} \cdot M \cdot s^{2} \\
P_{T f 41}=P_{T 21}+M \cdot s^{2} \cdot\left(P_{T 12} \cdot M \cdot s^{2}+2 \cdot P_{T 11}\right) \\
P_{T f 42}=P_{T 22}+P_{T 12} \cdot M \cdot s^{2}
\end{gathered}
$$

Values of Young modulus $\left(E_{l}\right)$ and material damping $\left(b_{1}\right)$ of the pipe can be obtained by making determinant of transfer matrix equal to zero, with $s$ (eigen number) chosen from measurement data.

\subsection{Water-filled pipe experiment}

Transfer matrix was formulated in a same way as in previous chapter. The process of formulation exceeds the scope of this contribution and as such is not covered. NavierStokes and continuity equations were applied so the final form allows us to enumerate values of bulk viscosity $\mu^{B}$ and speed of sound $c_{0}$ for water inside pipe. Material properties obtained by a manner covered in previous chapter were used so the Young modulus and damping of pipe are separate properties of structural part and the evaluated bulk viscosity and speed of sound are properties of fluid for a given simulated instance.

$$
\left(\begin{array}{l}
0 \\
0 \\
0 \\
0
\end{array}\right)=\mathbf{P}_{\text {Twater }} \cdot\left(\begin{array}{c}
\tilde{Q}_{0} \\
\tilde{p}_{0} \\
\tilde{Q}_{3} \\
\tilde{p}_{3}
\end{array}\right)
$$

Table 1. Mesh size independence test

\begin{tabular}{l|l|l} 
No. of elements & Frequency [Hz] & $\begin{array}{l}\text { Damping } \\
\text { parameter }[\mathrm{Hz}]\end{array}$ \\
\hline 1274 & 691,45 & $-5,2292$ \\
200 & 691,45 & $-5,2292$ \\
80 & 691,45 & $-5,2292$ \\
30 & 691,45 & $-5,2293$ \\
12 & 691,62 & $-5,2319$ \\
6 & 694,04 & $-5,2686$
\end{tabular}

\section{Simulation}

The computed model was only water-filled part of a pipe. Simulation was performed using ANSYS Mechanical program with Acoustics extension. This extension makes acoustic commands accessible in GUI of ANSYS FEM software. According to [7], there should be at least twelve linear elements of mesh per wavelength or six quadratic ones. Number of elements in a cross section of modelled cylinder is not as important as number of elements along the length of pipe, since the modal solution is longitudinal half wave.

The mesh size dependence analysis shows that few hundred quadratic elements is more than sufficient for accurate results. Table 1 contains values of simulation onemeter-long pipe meshed with quadratic elements HEX20.

Boundary conditions on the cylindrical surface as well as on the circular top and bottom is rigid wall. The rigid wall is default boundary condition in ANSYS Acoustics and means that acoustic velocity on a selected geometry is zero. In other words, acoustic velocity does not fluctuate and stays constant. Properties of fluid domain were density and viscosity (shear viscosity or volume viscosity), these parameters were same for all simulations. Speed of sound was regressed from an experimental data. The bulk viscosity varied in a range of measured bulk viscosities to encompass needed amount of values and compare them to the experiment.

\section{Custom code}

Software based on TMM (transfer matrix method) was used to determine if there is any difference in implementation of the bulk viscosity in ANSYS Acoustics and in custom code named FAchar. In Introduction to Acoustic [7], ANSYS states momentum equation with bulk viscosity $\mu^{B}$ Eq. (30).

$$
\rho \frac{\partial v_{a_{i}}}{\partial t}=-\nabla p_{a}+\left(\frac{4}{3} \mu+\mu^{B}\right) \cdot \nabla\left(\nabla \cdot v_{a_{i}}\right)
$$

And FAchar's bulk viscosity implementation in momentum equation is written as Eq. (31): 


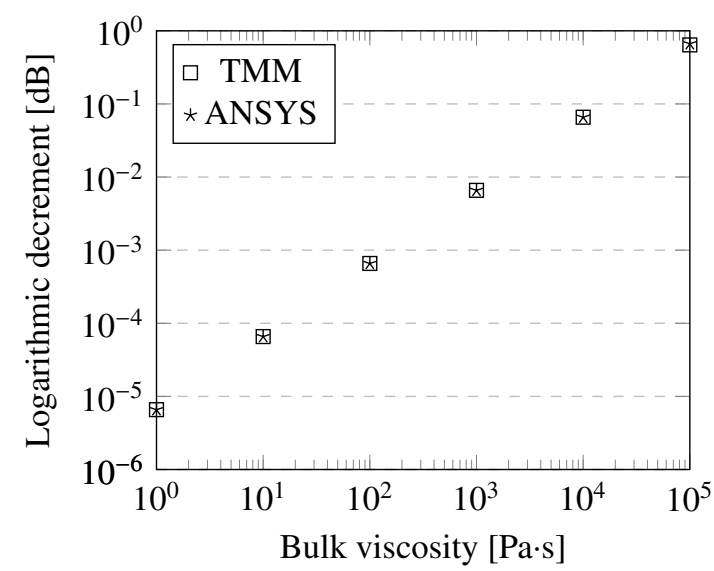

Fig. 2. ANSYS and custom code comparison

Table 2. ANSYS and custom code percental differences (ANSYS minus FAchar and divided by custom code value as a base)

\begin{tabular}{l|l|l} 
bulk v. [Pa·s] & freq. diff. [\%] & log. dec. diff. [\%] \\
\hline 1 & 0,00000017 & 0,138667 \\
10 & 0,00000017 & 0,013868 \\
100 & 0,00000017 & 0,001387 \\
1000 & 0,00000017 & 0,000085 \\
10000 & 0,00000018 & $-0,00547$ \\
100000 & 0,006218615 & $-0,56218$
\end{tabular}

$$
\begin{gathered}
\rho \frac{\partial v_{i}}{\partial t}=-\nabla p+\nabla \Pi_{i j} \\
\Pi_{i j}=\mu \frac{1}{2}\left(\frac{\partial v_{i}}{\partial x_{j}}+\frac{\partial v_{j}}{\partial x_{i}}\right)+\mu^{B} \frac{\partial x_{i} \cdot \partial v_{k}}{\partial x_{j} \cdot \partial x_{k}}
\end{gathered}
$$

The computation was performed for both cases on a model of one-meter-long pipe with diameter of $50 \mathrm{~mm}$. Speed of sound was $1500 \mathrm{~Pa} \cdot \mathrm{s}$, density $1000 \mathrm{~kg} \cdot \mathrm{m}^{-3}$ and bulk viscosities were six values from 1 to $100000 \mathrm{~m}^{2} \cdot \mathrm{s}^{-1}$.

It can be concluded from Figure 2 and Table 2 that with an exception at highest and lowest bulk viscosities the differences in data are very small so author concludes the implementations are sufficiently even and variations come from numeric methods used, discretization and precision.

\section{Results}

The results compared between the experiment and the simulation are damped frequency and logarithmic decrement of damping. Damped frequency values differ by a few percent, frequency obtained from the experiment and numer-
Table 3. Damped frequency comparison; pipe length $1 \mathrm{~m}$, diameter $23 \mathrm{~mm}$

\section{Experiment}

\begin{tabular}{l|l|l}
\hline $\begin{array}{l}\text { Sound speed } \\
{\left[\mathrm{m} \cdot \mathrm{s}^{-1}\right]}\end{array}$ & $\begin{array}{l}\text { Frequency } \\
{[\mathrm{Hz}]}\end{array}$ & $\begin{array}{l}\text { Analytic freq. } \\
{[\mathrm{Hz}]}\end{array}$ \\
\hline 1381,381578 & 695,418 & 690,691 \\
1383,582975 & 696,510 & 691,791 \\
1383,567324 & 696,510 & 691,784 \\
1383,572992 & 696,510 & 691,786 \\
1383,581693 & 696,510 & 691,791 \\
1383,58942 & 696,510 & 691,795
\end{tabular}

Acoustic simulation

\begin{tabular}{l|l|l}
\hline 1387,84 & 693,905 & 693,920 \\
1386,085 & 693,024 & 693,043 \\
1384,33 & 692,143 & 692,165 \\
1382,575 & 691,261 & 691,288 \\
1380,82 & 690,378 & 690,410
\end{tabular}

ical model is close to the theoretical frequency value. Analytical frequency can be calculated for a wave twice as long as a pipe. In Table 3 damped frequencies from experiment and simulation are compared to analytical value calculated for given speed of sound. Bigger difference of experimental values is expected result because there are more factors involved and those are omitted in simulation.

Logarithmic decrements show very similar trends in relation to bulk viscosity for all pipes but the differences are significantly bigger and up to ten percent. This is documented in Fig. 3, 4 and 5.

\section{Discussion}

Discrepancy in measurement and simulation data could be attributed to various causes. As already stated, in computation there is omission of pipe structure, so simulation has no influence of wall thickness, mass of flanges and measuring apparatus or damping in pipe material. In experiment a bending of pipe occurs and that is not implemented in simulation as well. Also, even small amount of air trapped in pipe can alter measured data [1]. Dissipation of energy caused by vibrations of pipe in air is another neglected effect. 


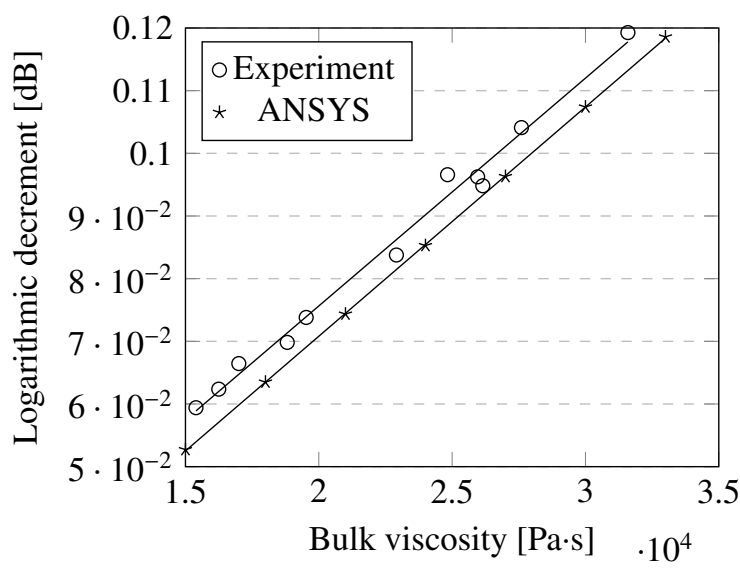

Fig. 3. ANSYS and experiment; pipe length $2 \mathrm{~m}$; diameter $32 \mathrm{~mm}$

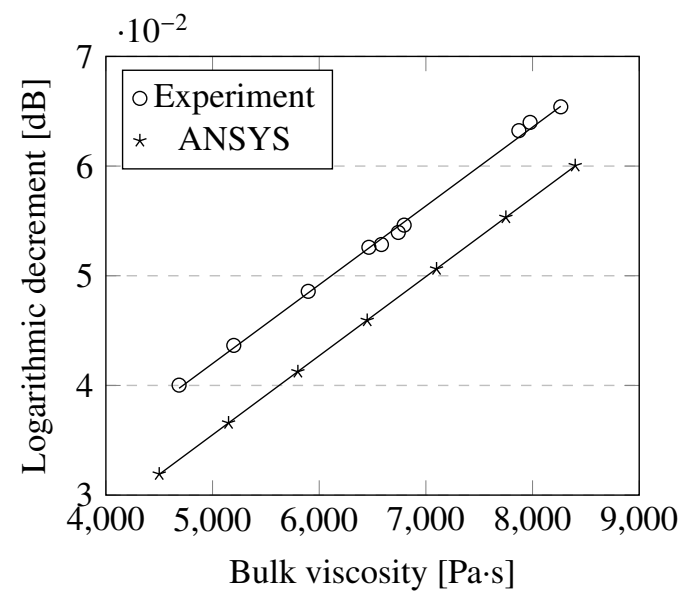

Fig. 4. ANSYS and experiment; pipe length $1 \mathrm{~m}$; diameter $32 \mathrm{~mm}$

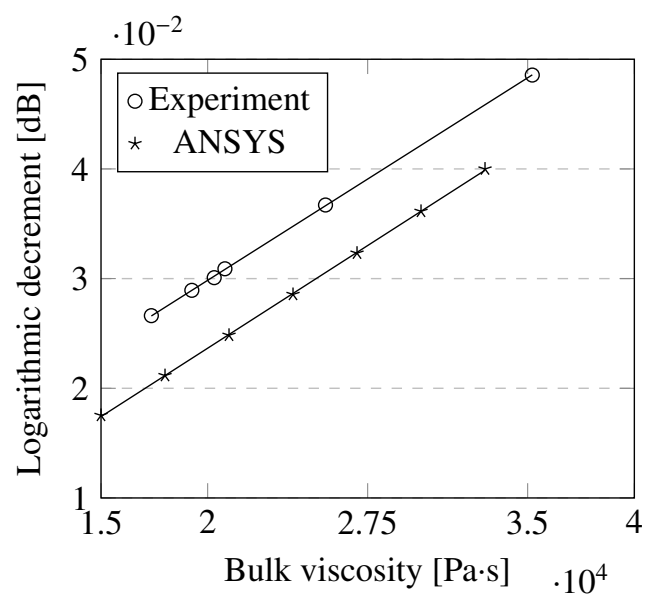

Fig. 5. ANSYS and experiment; pipe length $6 \mathrm{~m}$; diameter $42,5 \mathrm{~mm}$

\section{Conclusion}

Paper has described measurement of bulk viscosity and part of the mathematical model needed to evaluate data from obtained values of accelerations and pressure pulsations. Acquired bulk viscosities and eigen numbers were used with simulation. Ability to simulate model with bulk viscosity in ANSYS Acoustic and custom code is presented and results show conformity to measured values. Experiment and simulation vary, but given the simplification of computed model, it can still be considered good fit. Overall conclusion to be made is that acoustic simulation with bulk viscosity can yield results with acceptable precision.

Further work could focus on Fluid Structure interaction to take structural part into account and improve fidelity of modeled hydraulic system.

\section{Acknowledgements}

This work was supported by NETME Centre, regional R\&D centre built with the financial support from the Operational Programme Research and Development for Innovations within the project NETME Centre (New Technologies for Mechanical Engineering), Reg.no. CZ.1.05/2.1.00/ 01.0002 and, in the followup sustainability stage, supported through NETME CENTRE PLUS (LO1202) by financial means from the Ministry of Education, Youth and Sports under the "National Sustainability Programme I" and from the 2017 Science fund of Faculty of Mechanical engineering, Brno University of Technology project id.: FV 17-25.

\section{Nomenclature}

$\begin{array}{lll}b_{1} & {\left[\mathrm{~N} \mathrm{~s} \mathrm{~m}^{-1}\right]} & \text { damping of a pipe } \\ c_{0} & {\left[\mathrm{~m} \mathrm{~s}^{-1}\right]} & \text { speed of sound } \\ E_{1} & {[\mathrm{~Pa}]} & \text { stiffness constant of a pipe } \\ F & {[\mathrm{~N}]} & \text { force } \\ M & {[\mathrm{~kg}]} & \text { mass } \\ p & {[\mathrm{~Pa}]} & \text { pressure } \\ p_{a} & {[\mathrm{~Pa}]} & \text { acoustic pressure, amplitude of fluctuation } \\ \mathbf{P} & & \text { transfer matrix } \\ Q & {\left[\mathrm{~m}^{3} \mathrm{~s}^{-1}\right]} & \text { discharge } \\ s & {\left[\mathrm{~s}^{-1}\right]} & \text { parameter of Laplace transform } \\ S & {\left[\mathrm{~m}^{2}\right]} & \text { area } \\ S_{p} & {\left[\mathrm{~m}^{2}\right]} & \text { area of cross-section } \\ \mathbf{u} & & \text { state vector } \\ v & {\left[\mathrm{~m} \mathrm{~s}^{-1}\right]} & \text { velocity } \\ v_{a} & {\left[\mathrm{~m} \mathrm{~s}^{-1}\right]} & \text { acoustic velocity } \\ x & {\left[\mathrm{~m}^{2}\right.} & \text { dimension coordinate } \\ \rho & {\left[\mathrm{kg} \mathrm{m}^{-3}\right]} & \text { density } \\ \sigma & {[\mathrm{Pa}]} & \text { stress } \\ \mu & {[\mathrm{Pa} \mathrm{s}]} & \text { viscosity } \\ \mu^{B} & {[\mathrm{~Pa} \mathrm{~s}]} & \text { bulk viscosity } \\ \sim & & \text { variable after Laplace transform } \\ & & \end{array}$




\section{References}

1. HIMR D., HABÁN V.. Simulation of low pressure water hammer. IOP Conference Series: Earth and Environmental Science. 2010, 12, 012087-. DOI: 10.1088/1755-1315/12/1/012087. ISSN 1755-1315.

2. GRAVES R. E., ARGROW. B. M.. Bulk Viscosity: Past to Present. Journal of Thermophysics and Heat Transfer. 1999, 13(3), 337-342. DOI: 10.2514/2.6443. ISSN 0887-8722.

3. POCHYLÝ F., HABÁN V., FIALOVÁ S.. Bulk viscosity - constitutive equations. International Review of Mechanical Engineering, September 2011, Vol. 5 n. 6, pp. 1043-1051. ISSN: 1970-8734.

4. KORECK J., MAESS M., GAUL L.. AcousticStructure Simulation of Passive Fluid Pulsation Dampers, Fortschritte der Akustik: Plenarvorträge und Fachbeiträge der 33. Deutschen Jahrestagung für Akustik, DAGA 2007, Stuttgart. Berlin: DEGA, 2007. ISBN: 978-3-9808659-3-7.

5. EISINGER F. L., SULLIVAN R. E.. Vibration Fatigue of Centrifugal Fan Impeller Due to Structural-Acoustic Coupling and Its Prevention: A Case Study. Journal of Pressure Vessel Technology. 2007, 129(4), 771-. DOI: 10.1115/1.2767371. ISSN 00949930.

6. JONES P., KESSISSOGLOU N.. (2009). An evaluation of current commercial acoustic FEA software for modelling small complex muffler geometries: Prediction vs experiment. Annual Conference of the Australian Acoustical Society 2009 - Acoustics 2009: Research to Consulting.

7. ANSYS Inc., Introduction to acoustics, ANSYS Acoustics Lectures, 2016 